\title{
BMJ Open Cohort profile: the PHARMO Perinatal Research Network (PPRN) in the Netherlands: a population-based mother-child linked cohort
}

\author{
E. Houben (D) , ${ }^{1,2}$ L. Broeders, ${ }^{3}$ E.A.P. Steegers, ${ }^{2}$ R.M.C. Herings ${ }^{1,4}$
}

To cite: Houben E, Broeders L, Steegers E.A.P, et al. Cohort profile: the PHARMO Perinatal Research Network (PPRN) in the Netherlands: a population-based mother-child linked cohort. BMJ Open 2020;10:e037837. doi:10.1136/ bmjopen-2020-037837

- Prepublication history and additional material for this paper is available online. To view these files, please visit the journal online (http://dx.doi.org/10. 1136/bmjopen-2020-037837)

Received 26 February 2020

Revised 08 July 2020

Accepted 21 July 2020
Check for updates

\section{(C) Author(s) (or their} employer(s)) 2020. Re-use permitted under CC BY. Published by BMJ.

${ }^{1}$ PHARMO Institute for Drug Outcomes Research, Utrecht, The Netherlands

${ }^{2}$ Department of Obstetrics and Gynaecology, Erasmus MC, Rotterdam, The Netherlands ${ }^{3}$ Perined, Utrecht, The Netherlands

${ }^{4}$ Department of Epidemiology and Biostatistics, Amsterdam UMC, Amsterdam, The Netherlands

Correspondence to

E. Houben; pharmo@pharmo.nl

\section{ABSTRACT}

Purpose Observational population-based research is a very suitable non-invasive method for studies in the vulnerable populations of pregnant women and children. Therefore, the PHARMO Perinatal Research Network (PPRN) was set up as a resource for life course perinatal and paediatric research by linking population-based data from existing registrations.

Participants From 1999 to 2017, the PPRN captures approximately 542900 pregnancies of 387100 mothers ('Pregnancy Cohort'). Additionally, mother-child linkage is currently available for a quarter of these pregnancies ('Child Cohort'). The PPRN contains preconceptional information on maternal healthcare, as well as detailed pregnancy and neonatal data, extending into long-term follow-up and outcomes after birth for both mother and child up to nearly 20 years. It includes linked data from different primary and secondary healthcare settings. Findings to date Through record linkage of the Netherlands Perinatal Registry and the PHARMO Database Network, we have established a large population-based research network including data on demographics, medication use, medical conditions and details concerning labour, birth and neonatal outcomes. Here, we provide an overview of record types available from the PPRN, available database follow-up and pregnancy characteristics of the PPRN cohorts. The PPRN has been used for a number of different pharmacoepidemiological studies, for example, to confirm that preterm-born infants were more likely than full-term infants to be hospitalised or use medication. Similar long-term comparisons showed that children born following spontaneous preterm labour were at increased risk of neurodevelopmental and respiratory conditions. Most recently, the PPRN provided important evidence on the trends in use of potentially harmful medication during pregnancy.

Future plans The PPRN provides a unique and rich data set facilitating large-scale observational pharmacoepidemiological perinatal research. The patientlevel linkage of many different healthcare data sources allows for long-term follow-up of mother and child, with ongoing annual updates.

\section{INTRODUCTION}

Observational population-based research is a very suitable non-invasive method for studies in the vulnerable populations of pregnant women
Strengths and limitations of this study

- The main strength of the PHARMO Perinatal Research Network (PPRN) lies in the ongoing assembly of detailed, population-based, anonymised data from existing registrations which makes it an invaluable resource for life course perinatal and paediatric research.

- Evidence from clinical trials is often lacking behind for pregnant women and children, as researchers are often hesitant to include these subjects due to a variety of reasons. Hence, the PPRN provides a very suitable, non-invasive method that stays within the many risks and objections of studies in the vulnerable populations of pregnant women and children.

- The PPRN covers a considerable proportion of pregnancies from 1999 onwards that has been shown to reflect true estimates of the Dutch population captured in Netherlands Perinatal Registry (Perined), ensuring a high level of generalisability.

- Data collection periods and catchment areas vary between the linked databases and therefore the size of the study population depends on the databases included.

- Currently, we rely on probabilistic linkage methods as the number of records that include a social security number is currently too limited to allow for deterministic record linkage between the PHARMO Database Network and Perined.

and children. Therefore, the PHARMO Perinatal Research Network (PPRN) was set up as a resource for life course perinatal and paediatric research by linking population-based data from existing registrations. It was initiated around 2010 at the PHARMO Institute for Drug Outcomes Research in collaboration with Netherlands Perinatal Registry (Perined). At that time it was set up to study the relation between medication exposure during pregnancy and pregnancy outcomes, but the applications of the PPRN have extended considerably over the years, along with the continuous expansion of the underlying databases. 


\section{COHORT DESCRIPTION}

\section{Setting}

The PPRN is a unique linkage of the Perined and the PHARMO Database Network (PHARMO). With data collection starting in 1999, the linkage of these populationbased data sources facilitates large-scale observational pharmacoepidemiological perinatal research. It contains preconceptional information on maternal healthcare extending into long-term follow-up and outcomes after birth for both mother and child, with ongoing annual updates of the routinely collected data.

\section{Data sources}

Perined is a nationwide registry in which medical data around pregnancy and birth are included from pregnancies with a gestational age of at least 16 weeks (including terminated pregnancies and stillborns). ${ }^{1}$ It is a linked database combining medical registries from four professional groups that provide perinatal care: general practitioner, midwives, gynaecologists and neonatologists/paediatricians. Among the items reported are maternal demographics and medical conditions, pregnancy complications and details concerning labour, birth and neonatal outcomes. Linking the records is a complex operation-especially when it comes to records that originate from different data sets. Probabilistic linkage based on matching data is performed in the absence of unique identification of mother and/or child. There is a firm basis for deciding whether two records describe the same case or have a lot of resemblance. The threshold value for such a decision depends on the situation and is statistically substantiated. ${ }^{2}$ The established registry reflects virtually all deliveries in the Netherlands ( $\sim 99 \%$ agreement with the municipal administration), that is, including home as well as hospital births. The frequency of data collection and processing is four times a year. The average lag time of the data is half a year.

PHARMO is a population-based network of databases combining subnational data from different primary and secondary healthcare settings in the Netherlands. These different data sources, including data from general practices, inpatient and outpatient pharmacies, clinical laboratories, hospitals, the cancer registry, pathology registry and perinatal registry, are linked on a patient level through validated algorithms. ${ }^{3}$ Data are retrieved directly from the source, that is, the electronic medical records of the healthcare providers who agree to contribute to PHARMO. All patients registered at the contributing healthcare providers are included, unless the patient requested to opt out. To ensure the privacy of the data in the PHARMO Database Network, the collection, processing, linkage and anonymisation of the data are performed by the foundation 'Stichting Informatievoorziening voor Zorg en Onderzoek' (STIZON). STIZON is an independent ISO/IEC 27001 certified foundation, which acts as a trusted third party (TTP) between the data sources and the PHARMO Institute. Detailed information on the methodology and the validation of the used record linkage method can be found elsewhere. ${ }^{45}$ PHARMO covers approximately a quarter of the Dutch population and is shown to be representative of the Dutch population with regard to age and sex; however, data collection period, catchment area and overlap between data sources differ. The PHARMO databases are linked on an annual basis, the average lag time of the data is 1 year.

\section{Perined-PHARMO linkage}

STIZON also acts as a TTP for the linkage between Perined and PHARMO. This specific linkage is primarily based on the birth date of the mother and child, their gender and their zip codes. In case multiple possible links are established, these determinants are supplemented with hospital admission records around delivery as well as obstetrician or gynaecologist-prescribed medication. Furthermore, home codes that indicate mother and child live on the same address are used to verify established pairs and improve linkage specificity.

\section{Data collection}

From 1999 to 2017, the PPRN captures approximately 542900 pregnancies of 387100 mothers for which a PHARMO-Perined link could be established (ie, 'Pregnancy Cohort'). Additionally, an individual motherchild linkage is currently available for a quarter of these

\section{PHARMO Perinatal Research Network (PPRN)}

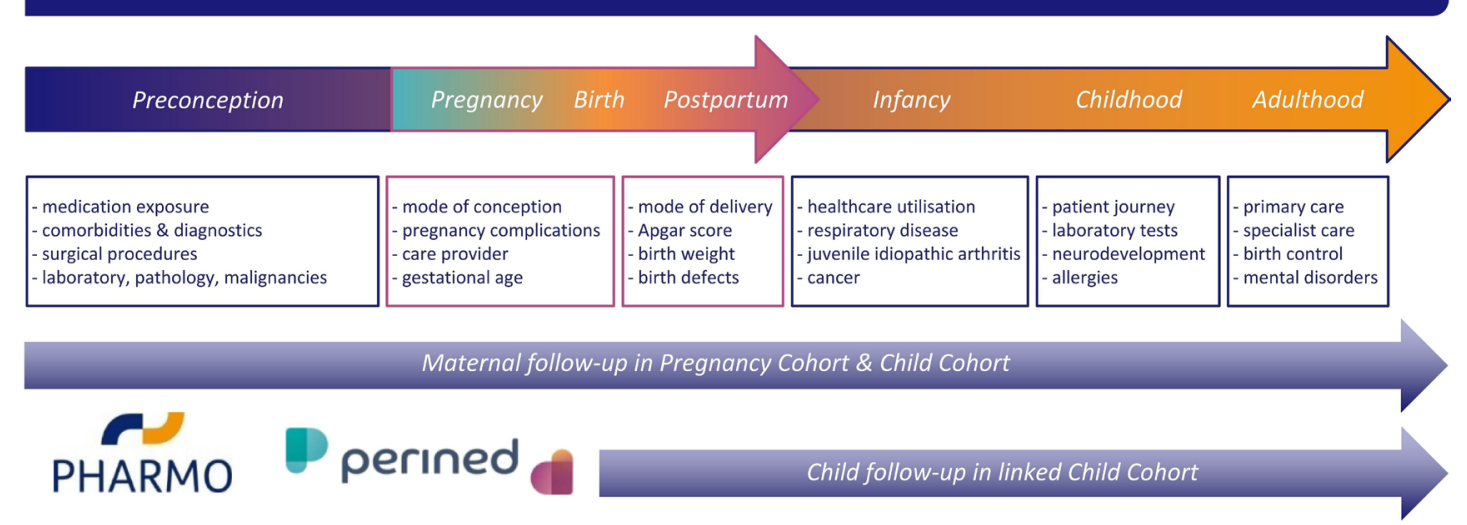

Figure 1 Schematic overview of data captured in the PHARMO-Perined linked PHARMO Perinatal Research Network (PPRN). 
Table 1 Overview of the record types available from the PPRN

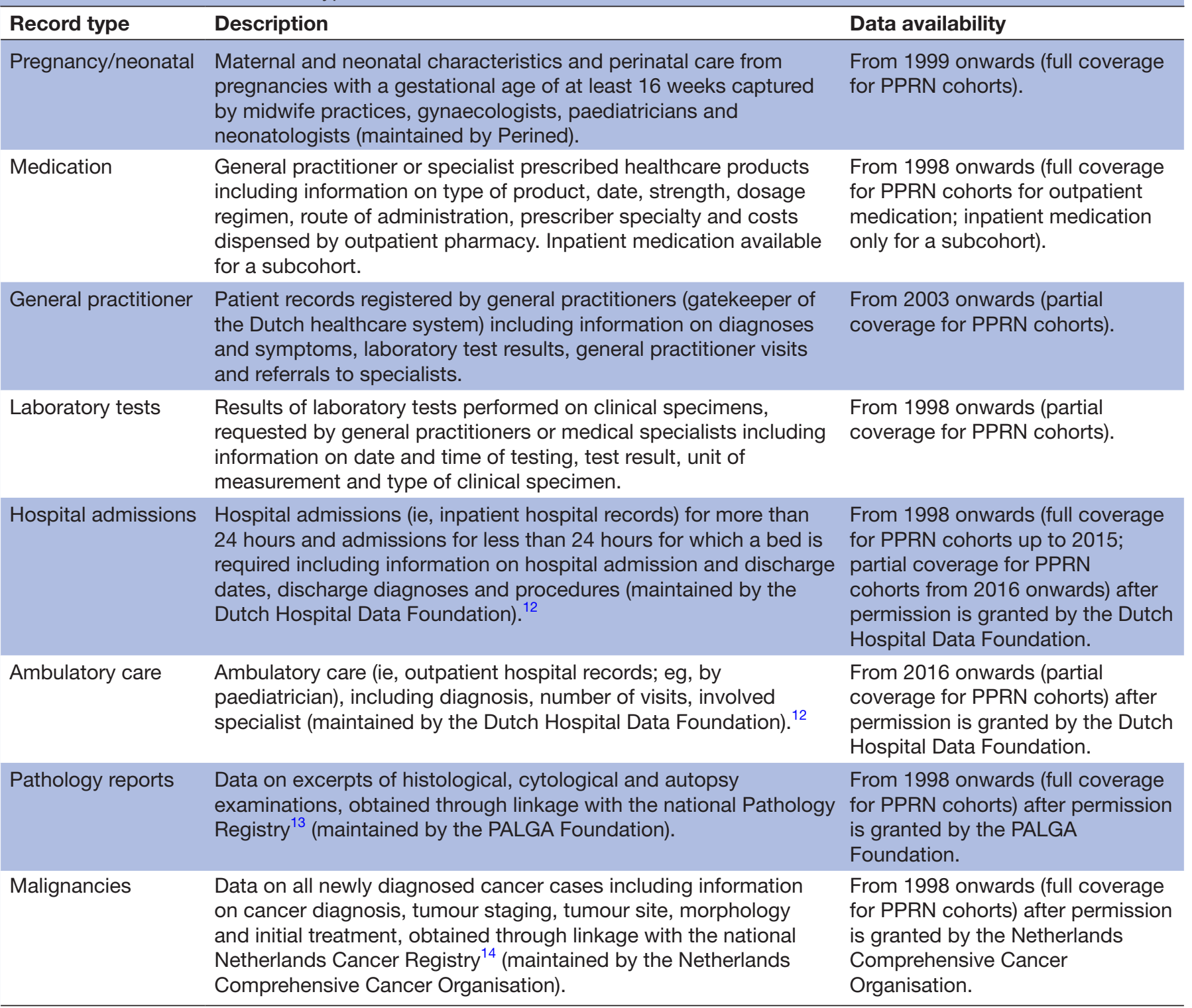

PPRN, PHARMO Perinatal Research Network.

pregnancies allowing subjects to be followed over time up to nearly 20 years after birth and studying associations with pregnancy or neonatal-specific outcomes (ie, 'Child Cohort'). A schematic overview of data captured in the PPRN for mothers and children and how these two cohorts inter-relate is included in figure 1 and table 1 . Further characterisation of the PPRN is included in table 2, including the total Perined population as a reference, considering that only a subsample of the Netherlands is represented by the PHARMO Database Network. Figure 2 presents the number of pregnancies included in the Pregnancy Cohort and Child Cohort by calendar year. Details on the available database follow-up for the children included in the Child Cohort are presented in figure 3, with end of follow-up defined by either end of database registration (ie, the patient moves out of the PHARMO catchment area), death or end of study period (31 December 2018), whichever occurred first.

\section{Patient and public involvement}

No patients were involved in the described linkage between existing registries providing an anonymous data set.

\section{FINDINGS TO DATE}

Through record linkage of Perined and PHARMO, we have established a large population-based research network including data on demographics, medication use, medical conditions, pregnancy complications and details concerning labour, birth and neonatal outcomes. The PPRN has been used for a number of different pharmacoepidemiological studies (see online supplemental appendix 1 for a citation list of work published on the PPRN). Its applicability can be centred on the mother, the child or both (ie, assessing 
Table 2 Pregnancy characteristics in the Pregnancy Cohort and the Child Cohort of the PPRN

PHARMO Perinatal Research Network

Perined 1999(PPRN) 1999-2017 2017 (reference)

\begin{tabular}{|c|c|c|c|}
\hline & Pregnancy Cohort & Child Cohort & Total population \\
\hline Pregnancies (n) & $\sim 542900$ & $\sim 126200$ & $\sim 3200000$ \\
\hline Mothers (n) & $\sim 387100$ & $\sim 101400$ & - \\
\hline \multicolumn{4}{|l|}{ Maternal characteristics } \\
\hline Age at delivery (mean $\pm S D$; years) & $31.0 \pm 4.8$ & $30.8 \pm 4.7$ & $31.0 \pm 4.9$ \\
\hline Nulliparous (\%) & 46 & 57 & 47 \\
\hline Dutch ethnicity (\%) & 79 & 84 & 79 \\
\hline Database history before delivery (mean $\pm \mathrm{SD}$; years) & $6.0 \pm 4.3$ & $6.1 \pm 4.3$ & - \\
\hline Database follow-up after delivery (mean $\pm S D$; years) ${ }^{*}$ & $7.9 \pm 5.0$ & $7.7 \pm 4.7$ & - \\
\hline \multicolumn{4}{|l|}{ Infant characteristics } \\
\hline Male sex (\%) & 51 & 53 & 51 \\
\hline Gestational age at birth (mean $\pm S D$; weeks) & $39.1 \pm 3.5$ & $39.2 \pm 2.7$ & $39.3 \pm 2.3$ \\
\hline Preterm birth (\%) & 8 & 7 & 8 \\
\hline Multiple birth (\%) & 2 & $<0.5$ & 4 \\
\hline Database follow-up after birth (mean $\pm S D$; years) ${ }^{*}$ & $7.8 \pm 4.7$ & $7.8 \pm 4.7$ & - \\
\hline
\end{tabular}

${ }^{*}$ Current censoring: 31 December 2018.

the association between maternal characteristics and child outcomes). As an example, medication use during first year of life and hospital admission rates have been assessed and compared between premature and term infants. ${ }^{6}$ Pretermborn infants were up to two times more likely than full-term infants to be hospitalised or use medication, especially related to respiratory disease. Similar long-term comparisons of morbidities and healthcare utilisation have been made which showed that children born following spontaneous preterm labour (irrespective of gestational age at delivery) were at increased risk of neurodevelopmental and respiratory conditions compared with those from full-term labour pregnancies. ${ }^{7}$ Most recently, data from the PPRN have been used to determine population-based trends over the last two decades in the use of potentially harmful medication among pregnant women. ${ }^{8}$

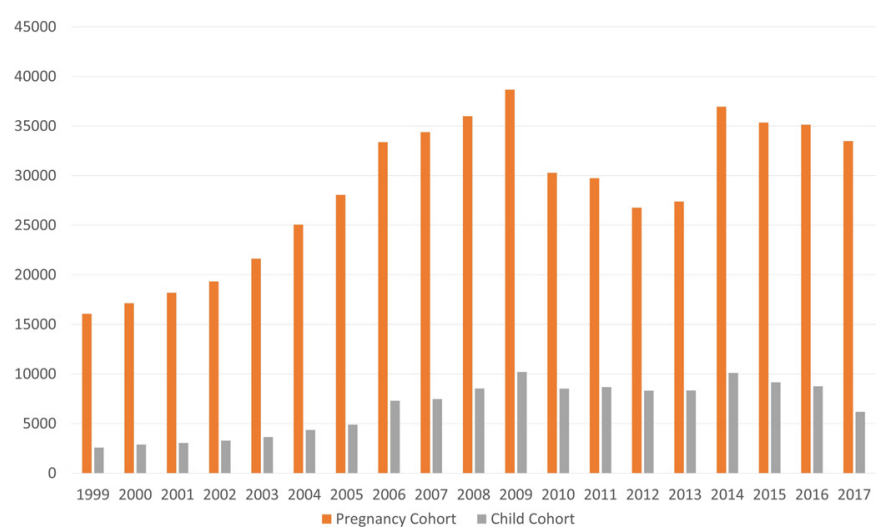

Figure 2 Number of pregnancies included in the Pregnancy Cohort and Child Cohort by calendar year.

\section{STRENGTHS AND LIMITATIONS}

The main strength of the PPRN lies in the ongoing assembly of detailed, population-based, anonymised data from existing registrations which makes it an invaluable resource for life course perinatal and paediatric research. Evidence from clinical trials is often lacking behind for pregnant women and children, as researchers are often hesitant to include these subjects due to a variety of reasons, including the fear of harm to the fetus and threat of legal liability. ${ }^{9} 10$ Therefore, the PPRN provides a very suitable, non-invasive method that stays within the many risks and objections of studies in the vulnerable populations of pregnant women

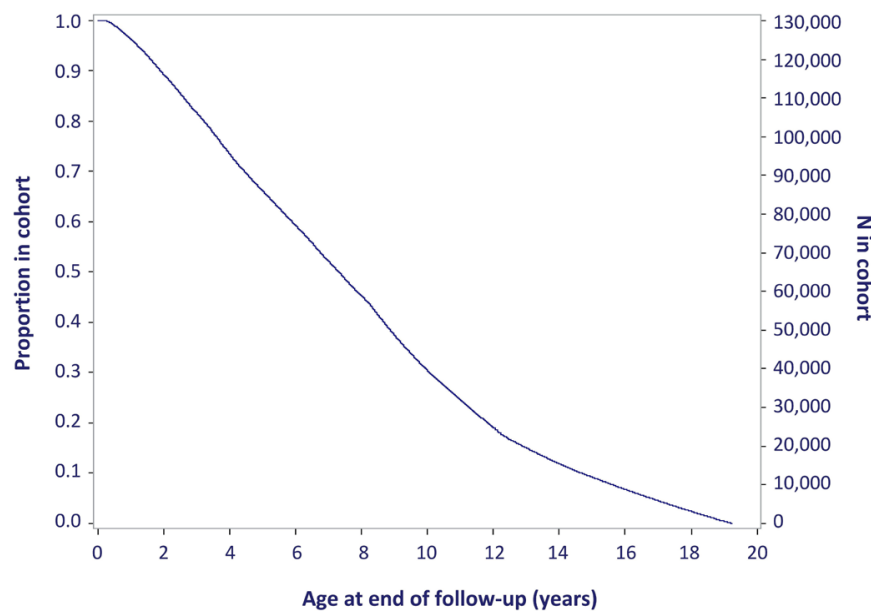

Figure 3 Proportion and number of children included in the PHARMO Perinatal Research Network (PPRN) Child Cohort, with age in years at end of follow-up (current censoring: 31 December 2018). 
and children. The PPRN covers a considerable proportion of pregnancies from 1999 onwards that has been shown to reflect true estimates of the Dutch population captured in Perined, ${ }^{11}$ ensuring a high level of generalisability. The patient-level linkage of many different healthcare data sources provides a very rich data set allowing long-term follow-up of mother and child, with data continuously being collected.

The PPRN brings together data from various sources. Data collection periods and catchment areas vary between these databases and therefore the size of the study population depends on the databases included. The 542900 pregnancies linked in the data cut up to 2017 allow for assessment of drug use during the 9-month preconception, pregnancy and 9-month postpartum periods. Inclusion of other databases (eg, general practitioner records or hospital admissions) will reduce the cohort size. As with any database, identification of medical events is limited to data that are captured as part of the medical records or other linked data sources in daily clinical practice. These data are not primarily collected for research purposes and rely on appropriate diagnostic coding. Also, the lag time for the PHARMO-Perined linked data to become available is currently approximately 1 year. Furthermore, the number of records that include a social security number is currently too limited to allow for deterministic record linkage between PHARMO and Perined. This availability is steadily increasing and will in the future improve the ability to differentiate between siblings in case of multiple birth pregnancies, which are now under-represented in the Child Cohort. The current linkage methods particularly gain a high specificity, and including these unique patient identifiers the sensitivity is expected to increase further as well. The seeming under-representation of multiple births in the Pregnancy Cohort is caused by the fact that the presented reference proportion for the total Perined population includes all pregnancies (including terminated pregnancies and stillborns); however, comparisons by gestational age indeed show agreement between the two (data not presented). Furthermore, the higher proportion of nulliparous women in the Child Cohort compared with the other two cohorts is mainly influenced by families more often moving houses shortly after delivery of a second child compared with their first child, and due to the new address it is less likely that the child can be traced back in the PHARMO Database Network.

\section{COLLABORATION}

Access to the PPRN is, by governance regulations of the data collection and contractually agreed between the PHARMO Institute and Perined, restricted to researchers of the PHARMO Institute, Perined and academic affiliates. Academic affiliates from universities, hospitals or other research institutes are encouraged to apply for access to the anonymised data for scientific study purposes. The data are handled in accordance with data protection, privacy regulations and ISO certification schemes. Each data request is checked against these policies and requires permission of the applicable compliance and privacy boards of both PHARMO and Perined. Permission to external databases is requested from the database holders (eg, Dutch Hospital Data Foundation or PALGA Foundation) on a project basis. As it concerns database research with anonymous data, no Institutional Review Board or ethics committee approval is required. An overview of the variables included in the different databases, the terms and conditions and data application forms are available on http://pharmo.nl/whatwe-have/data-request-PHARMO/ and (in Dutch) www. perined.nl/registratie/faciliteren-onderzoek. Data sets are processed in SAS version 9.4 (SAS Institute), but can be converted to other data formats. Only a $10 \%$ subsample of the requested data can be downloaded by the researcher from a secure FTP server; access to the full data set can be granted to researchers guesting at the PHARMO office.

Contributors EH and RH had full access to all of the data in the study and take responsibility for the integrity of the data and the accuracy of the data analysis. EH, LB, EAPS and RH contributed to the plan and design of the study. EH performed the data analyses and drafted the manuscript and was in charge of the study planning. EH, LB, EAPS and RH contributed to the interpretation of the results and critical revision of the manuscript for important intellectual content and approved the final version of the manuscript. EH and RH are the guarantors of this paper.

Funding The authors have not declared a specific grant for this research from any funding agency in the public, commercial or not-for-profit sectors.

\section{Competing interests None declared.}

Patient and public involvement Patients and/or the public were not involved in the design, or conduct, or reporting, or dissemination plans of this research.

\section{Patient consent for publication Not required.}

Provenance and peer review Not commissioned; externally peer reviewed.

Data availability statement Data are available upon reasonable request. Requests for sharing study data must be made on specific grounds, either (1) with the aim of corroborating the study results in the interest of public health or (2) in the context of an audit by a competent authority. Sufficient information needs to be provided to confirm that the request is made for one of the above-mentioned purposes, including a sound justification and, in case of a request with a view to corroborate study results, a protocol on the research for which the data will be used or a plan for quality control checks, as applicable.

Open access This is an open access article distributed in accordance with the Creative Commons Attribution 4.0 Unported (CC BY 4.0) license, which permits others to copy, redistribute, remix, transform and build upon this work for any purpose, provided the original work is properly cited, a link to the licence is given, and indication of whether changes were made. See: https://creativecommons.org/ licenses/by/4.0/.

ORCID iD

E. Houben http://orcid.org/0000-0002-9173-3335

\section{REFERENCES}

1 Grote Lijnen 1999-2012. Stichting perinatale registratie Nederland, 2013.

2 Méray N, Reitsma JB, Ravelli ACJ, et al. Probabilistic record linkage is a valid and transparent tool to combine databases without a patient identification number. J Clin Epidemiol 2007;60:883.e1-883. e11.

3 Kuiper JG, Bakker M, Penning-van Beest FJA, et al. Existing data sources for clinical epidemiology: the PHARMO database network. Clin Epidemiol 2020;12:415-22.

4 Herings R, Pedersen L. Pharmacy-based medical record linkage systems. In: Strom B, Kimmel S, Hennessy S, eds. Pharmacoepidemiology. 5th edn. John Wiley \& Sons, Ltd, 2012: $270-86$. 
5 van Herk-Sukel MPP, van de Poll-Franse LV, Lemmens VEPP, et al. New opportunities for drug outcomes research in cancer patients: the linkage of the Eindhoven cancer registry and the PHARMO record linkage system. Eur J Cancer 2010;46:395-404.

6 Houweling LMA, Bezemer ID, Penning-van Beest FJA, et al. First year of life medication use and hospital admission rates: premature compared with term infants. J Pediatr 2013;163:61-6.

7 Houben E, Smits E, Pimenta JM, et al. Increased risk of morbidities and health-care utilisation in children born following preterm labour compared with full-term labour: a population-based study. J Paediatr Child Health 2019;55:446-53.

8 Houben E, Te Winkel B, Steegers EAP, et al. Dutch trends in the use of potentially harmful medication during pregnancy. $\mathrm{Br} \mathrm{J}$ Clin Pharmacol 2020;157
9 Blehar MC, Spong C, Grady C, et al. Enrolling pregnant women: issues in clinical research. Womens Health Issues 2013;23:e39-45.

10 Sheffield JS, Siegel D, Mirochnick M, et al. Designing drug trials: considerations for pregnant women. Clin Infect Dis 2014;59:S437-44.

11 Perined. Perinatale Zorg in Nederland 2016. Perined: Utrecht, 2018.

12 Dutch hospital data Foundation. Available: https://www.dhd.nl/ Paginas/home.aspx

13 Palga Foundation. The nationwide network and registry of histo- and cytopathology in the Netherlands, 2018. Available: http://www.palga. $\mathrm{nl} /$ [Accessed 17 Apr 2018].

14 Netherlands Comprehensive Cancer Organisation. Netherlands cancer registry, 2018. Available: https://www.iknl.nl/ [Accessed 17 Apr 2018]. 